\title{
PRODUCTIVIDAD Y DISTRIBUCIÓN DEL INGRESO: IMPLICACIONES SOBRE EL SALARIO MÍNIMO COLOMBIANO, 2001-2016*
}

Daniel Francisco Ossa Hernándeza

"DOI: https://doi.org/10.18601/01245996.v20n39.10. Este artículo es resultado de una investigación del Grupo de Socioeconomía, Instituciones y Desarrollo (GSEID) del Centro de Investigaciones para el Desarrollo (CID) de la Universidad Nacional de Colombia. Recepción: 24-10-2017, modificación final: 22-01-2018, aceptación: 11-05-2018. Sugerencia de citación: Ossa H., D. F. (2018). Productividad y distribución del ingreso: implicaciones sobre el salario mínimo colombiano, 2001-2016. Revista de Economía Institucional, 20(39), 231-255.

a Economista, investigador del GSEID, [dafossahe@unal.edu.co]. 


\section{Productividad y distribución del ingreso: implicaciones sobre el salario mínimo colombiano, 2001-2016}

Resumen. Este trabajo revisa la discusión sobre el método más adecuado para calcular la productividad. Toma en cuenta la productividad total de los factores, la productividad del capital y la productividad laboral, así como sus interpretaciones, alcances y relaciones. Además, analiza el impacto de esta discusión en la evolución del salario mínimo colombiano durante el periodo 2001-2016. Por último, discute qué relación existe entre el crecimiento de la productividad y la distribución del ingreso entre el capital y el trabajo, proponiendo una crítica a la visión neoclásica.

Palabras clave: salario mínimo, productividad, PTF, productividad laboral, distribución del ingreso; JEL: J24, J30, O47, B40, E24.

\section{Productivity and income distribution: Implications for the Colombian minimum wage, 2001-2016}

Abstract. This paper reviews the discussion on the most appropriate method for measuring productivity. It takes into account the TFP, the productivity of capital, and labor productivity, as well as its interpretations, scope, and relations. Additionally, it analyzes the impacts of this discussion on the evolution of the Colombian minimum wage over the period 2001-2016. Finally, it discusses the relationship between the growth of productivity and the distribution of income between capital and labor, proposing a critique of the neoclassical perspective.

Keywords: Minimum salary, productivity, TFP, labor productivity, income distribution; JEL: J24, J30, O47, B40, E24.

\section{Produtividade e distribuição da renda: implicações sobre o salário-mínimo co- lombiano, 2001-2016}

Resumo. Este trabalho revisa a discussão sobre o método mais adequado para calcular a produtividade. Leva em consideração a produtividade total dos fatores, a produtividade do capital e a produtividade profissional, bem como suas interpretações, alcances e relações. Além disso, analisa o impacto da discussão na evolução do salário-mínimo colombiano durante 2001-2006. Por último, discute quais relações existem entre o crescimento da produtividade e a distribuição da renda entre o capital e o trabalho, propondo uma crítica à visão neoclássica.

Palavras-chaves: salário-mínimo, produtividade, PTF, produtividade profissional, distribuição do rendimento; JEL: J24, J30, O47, B40, E24. 
$\mathrm{C}$ on el cambio de patrón de acumulación capitalista a comienzos de la década de los años setenta, y el comienzo de la aplicación de políticas neoliberales como su expresión, la relación entre el capital y el trabajo cambió drásticamente a nivel mundial. Parte de esta modificación se evidencia en la tendencia de los salarios a crecer menos que la productividad, a estancarse o incluso a disminuir en términos reales en algunos países. En Estados Unidos, la brecha entre el crecimiento de los salarios reales y el de la productividad laboral comenzó a crecer en forma sostenida desde 1979 (Shaikh, 2016, pp. 60-61), y llegó a más del 10\% en promedio en las 35 principales economías entre 1999 y 2013 (Wolff, 2016, p. 71). De modo que no es una tendencia que se observe en un solo país. Lapavitsas (2013, pp. 244-245) muestra, además, que dicha brecha está acompañada de una reducción de la participación del trabajo en el producto bruto en Estados Unidos, Japón, Reino Unido y Alemania.

En este contexto, cabe preguntar si esto ha ocurrido en Colombia y qué formas ha tomado. Se podría pensar que no ha ocurrido en el caso del salario mínimo porque la Ley 278 de 1996, que regula la negociación anual del salario mínimo, establece la productividad como uno de los criterios para aumentarlo. Este cálculo lo hace la Sub-Comisión de Productividad, una primera mesa que se reúne en el marco de las negociaciones del salario mínimo y, que al menos en los últimos 16 años, ha calculado la productividad a partir del modelo de Solow y establecido la variación anual de la productividad total de los factores (PTF).

El problema es que, desde hace varias décadas, una amplia literatura argumenta que la PTF no se puede equiparar a la productividad. De ser así, los aumentos del salario mínimo en este periodo se han basado en un criterio de ajuste erróneo. Este artículo revisa la discusión sobre el cálculo de la productividad, analiza sus efectos sobre la evolución del salario mínimo durante el periodo 2001-2016 y su impacto en la distribución del ingreso entre capital y trabajo. Cabe señalar que algunos trabajos, como el de Valle (1991), discuten la manera de calcular la productividad, y la relación entre PTF y productividad laboral, aquí se intenta profundizar la discusión y exponer sus implicaciones para la fijación del salario mínimo.

\section{EL CONTEXTO DE LA DISCUSIÓN}

La Ley 278 de 1996 creó la “comisión permanente de concertación de políticas salariales y laborales" aún vigente, una de cuyas funciones 
es "fijar de manera concertada el salario mínimo de carácter general, teniendo en cuenta que se debe garantizar una calidad de vida digna para el trabajador y su familia”. El artículo 8 señala que sus decisiones serán adoptadas por consenso, y si no se logra un consenso el gobierno lo determinará teniendo en cuenta la meta de inflación del año siguiente, la productividad acordada por el comité tripartito de productividad y la contribución de los salarios al ingreso nacional.

La Sentencia C-815 de 1999 de la Corte Constitucional modificó dicha ley. Estableció que, además de los parámetros señalados, se debe tener en cuenta la inflación real del año que culmina; lo cual significa que el salario mínimo no puede disminuir en términos reales de un año a otro.

La decisión de la Corte Constitucional llevó a que desde 2000 el salario mínimo haya aumentado al menos al ritmo de la inflación. Pero como es una negociación política, un aumento superior a la inflación depende de la fuerza de cada sector en la mesa. Por esa razón y debido al poco poder de negociación de las centrales obreras, el gobierno y los empresarios han dado prioridad a las propuestas de aumentos basados en la inflación del año corrido y la variación de la productividad. Así, en la mayoría de estos años los aumentos anuales se han basado en la suma de estos dos datos. Es posible que esta regla haya sido el criterio principal, porque para la teoría ortodoxa los aumentos salariales basados en las inflación más la productividad no ejercen presiones inflacionarias (Cortés, 2010, p. 123).

En este contexto de negociación, en los últimos 16 años la productividad se ha calculado a partir del residuo de Solow, es decir, de la PTF. Al comparar las variaciones anuales de la PTF y del salario mínimo real se observa que la PTF se tuvo en cuenta para decretar los aumentos en la mayoría de los años, pero que en los años pico (2004, 2006 y 2007) no se tomó en cuenta en su totalidad, pues el aumento del salario mínimo en términos reales fue menor, como muestra la gráfica 1. Cabe señalar que en esos tres años no hubo acuerdo en la mesa y el gobierno decretó el aumento (Rivera, 2014, p. 10).

No obstante, la gráfica 2 muestra que en este periodo los aumentos del salario mínimo, en forma acumulada, han estado por encima de la PTF. Esto indicaría que la tendencia observada a nivel mundial de un crecimiento de los salarios inferior al de la productividad no se observa en el caso del salario mínimo colombiano durante el periodo 2001-2016.

Pero como se mostrará más adelante, la PTF no es un indicador de productividad. Para abordar esta discusión es necesario tratar los 
orígenes y las bases de la teoría que sustenta esta forma de medir la productividad. Ese es el tema de la siguiente sección.

Gráfica 1

PTF y salario mínimo real

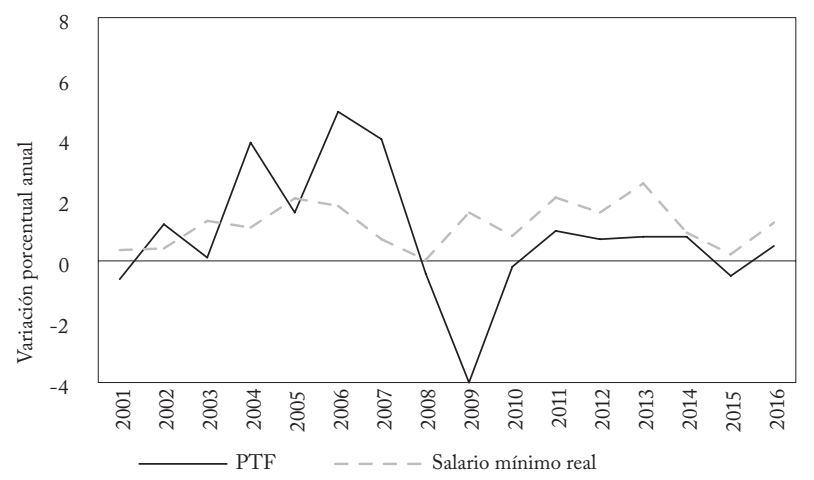

Fuente: DNP, elaboración propia.

Gráfica 2

Índice de PTF y salario mínimo real $(2000=100)$

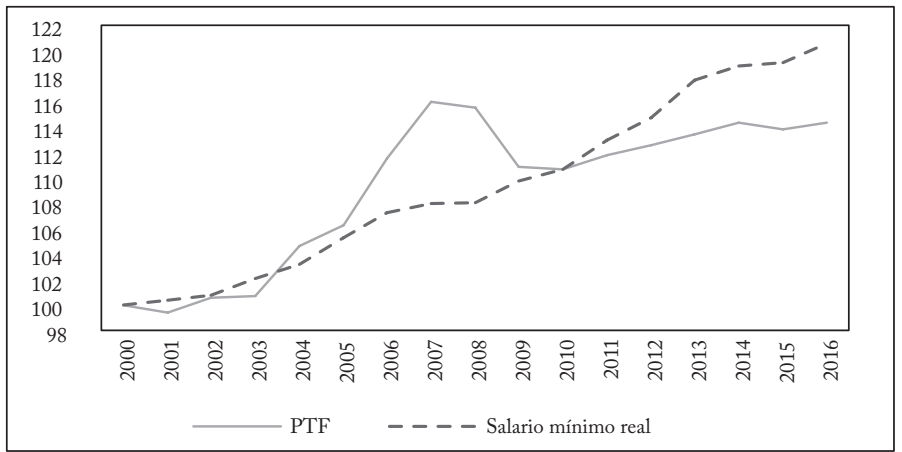

Fuente: DNP, elaboración propia.

\section{LA FUNCIÓN COBB-DOUGLAS, EL MODELO DE SOLOW YLA PTF}

La función Cobb-Douglas, base del modelo de Solow, fue presentada por Charles Cobb y Paul Douglas en su artículo de 1928. Su objetivo era representar la producción de una empresa o de una economía mediante una función matemática, es decir, relacionar la cantidad de producto final $(Y)$ con las cantidades de trabajo $(L)$ y capital $(K)$, como muestra la ecuación (1). La idea era analizar la incidencia relativa del 
trabajo y del capital en la producción, y si los ingresos del capital y del trabajo corresponden a esas condiciones técnicas de producción. $Y=f(L, K)$

Una de las intenciones de los autores era establecer una función de producción que permitiera, usando datos reales, corroborar la teoría de la distribución del producto propuesta por la teoría neoclásica, que explica la distribución entre capital y trabajo por el aporte marginal de cada factor.

Ese trabajo fue motivado porque Douglas advirtió que en Estados Unidos la distribución del producto entre capital y trabajo se había mantenido constante durante varios años. Los datos permitían calcular los productos marginales de cada factor, y así comprobar la hipótesis de la teoría neoclásica. De modo que la función debía satisfacer las ecuaciones (2) y (3), donde (w) es el salario real, $\left(\frac{\partial Y}{\partial L}\right)$ la productividad marginal del trabajo, $(r)$ la tasa de ganancia real, $\left(\frac{\partial Y}{\partial K}\right)$ la productividad marginal del capital, y $(\alpha)$ y $(\beta)$ la elasticidad del producto con respecto al trabajo y al capital, respectivamente.

$$
\begin{aligned}
& w=\left(\frac{\partial Y}{\partial L}\right)=\propto\left(\frac{\partial Y}{\partial K}\right) \\
& r=\left(\frac{\partial Y}{\partial K}\right)=\beta\left(\frac{Y}{K}\right)
\end{aligned}
$$

La función de producción Cobb-Douglas se representa en la expresión (4), que cumple las condiciones anteriores, suponiendo que la distribución del producto entre capital y trabajo se mantiene constante. Esta expresión incluye además el coeficiente $(A)$, que es independiente del trabajo y del capital, y capta los efectos de cualquier fuerza que afecte la producción que no esté representada en los datos (Cobb y Douglas, 1928, p. 155).

$Y=A L^{\alpha} K^{\beta}$

Para comprobar la hipótesis neoclásica de la distribución del producto era necesario estimar las elasticidades marginales del producto con respecto a cada factor, mediante una regresión, y luego comparar el resultado con las participaciones reales de cada factor en el producto. $\mathrm{Si}$ ambas resultaban significativamente iguales se comprobaba la hipótesis neoclásica.

Los autores creyeron que había comprobado su hipótesis porque estos dos resultados fueron muy cercanos. Como señala Douglas (1976, p. 914), se consideró que este resultado validaba la teoría neoclásica y refutaba la teoría marxista de la distribución del producto. 
Esto significa que se podía rechazar la idea de explotación, central en la obra de Marx para explicar el proceso de acumulación capitalista, si se comprobaba que el capital es productivo en sí mismo. Intentaban, entonces, demostrar, con datos reales, que la ganancia -el ingreso del capital- tenía estrecha relación con su productividad marginal, y que los salarios, la porción del producto correspondiente al trabajo, se explicaba por su productividad marginal ${ }^{1}$.

Cobb y Douglas encontraron, además, que la suma de los valores de $(\alpha)$ y $(\beta)$ se acercaba a 1 , y concluyeron que la economía en estudio presentaba rendimientos constantes a escala. Esto significa que un aumento del capital y del trabajo en cierta proporción genera un aumento de la cantidad de producto en la misma proporción. Así, la expresión (4) se podía reescribir haciendo que $(\beta)=(1-\alpha)$.

$Y=A L^{\alpha} K^{1-\alpha}$

Aunque el trabajo fue muy criticado en un comienzo (Felipe y McCombie, 2013, pp. 139-142), después fue ampliamente acogido, en especial en las teorías neoclásicas del crecimiento económico.

E1 modelo de Solow, presentado por su autor en 1956, se puede representar inicialmente como una función en la que el producto $(Y)$ depende del trabajo $(L)$ y el capital $(K)$. Es necesario resaltar que esta relación se presenta en unidades físicas, pues el modelo solo incluye un producto, que además representa el capital.

Solow extiende el modelo introduciendo la posibilidad de cambio técnico, suponiendo que no altera las tasas marginales de sustitución entre capital y trabajo. Es decir, solo aumenta el producto sin alterar las proporciones de factores en la función de producción. Por ello lo llama cambio técnico neutral $(A)$.

$Y=A f(L, K)$

En su artículo de 1957, Solow amplió el alcance del modelo. Su objetivo era ahora separar las variaciones del producto per cápita entre las atribuibles al cambio técnico y las relacionadas con la disponibilidad de capital por trabajador. Es decir, establecer qué parte del crecimiento económico se debía a aumentos de la disponibilidad de capital y qué parte al cambio técnico. Este último no entendido necesariamente

${ }^{1}$ Aquí cabe una precisión. Cobb y Douglas señalan que sus resultados no justifican el orden social y económico existente. Es decir, que no llevan a concluir que el capital deba o no ser privado, pues el hecho de que el capital sea productivo no significa que los capitalistas lo sean. Así, se puede ser socialista, comunista o individualista y la teoría encaja con cualquiera de esas visiones (Cobb y Douglas, 1928, pp. 163-164). 
como desarrollo tecnológico, sino como "cualquier tipo de cambio en la función de producción” (Solow, 1957, p. 312).

Para ello presentó el modelo anterior, esta vez sin definir en forma explícita una función de producción específica. Pero como supone que, a través del tiempo, cada factor recibe un pago constante que corresponde a su productividad marginal, cabe pensar que es de tipo Cobb-Douglas, que adopta los mismos supuestos.

Además, en ambos artículos Solow supuso rendimientos constantes a escala en la función de producción, es decir, que era homogénea de primer grado ${ }^{2}$. A este respecto, en el artículo de 1956 dice que los "rendimientos constantes a escala parecen ser el supuesto natural para hacer una teoría de crecimiento" (1956, p. 67). Y en el de 1957, que "si todos los factores de producción se clasifican como capital o trabajo, las cifras disponibles muestran que $w_{L}$ y $w_{K}$ (las participaciones relativas del capital y el trabajo) suman 1" (1957, p. 313).

Así, el modelo se representa mediante la ecuación (5). La diferencia radica en que el parámetro $(A)$ tiene una interpretación distinta a la de Cobb y Douglas (1928), pues ahora se entiende como cambio técnico neutral, y en la literatura luego se conocerá como productividad total de los factores. Derivando la ecuación (5) con respecto al tiempo y reorganizando términos se llega a la ecuación (7), donde $\left(\frac{\dot{A}}{A}\right)$ representa la tasa de crecimiento de la PTF. En el otro lado de la ecuación $\left(\frac{\dot{Y}}{Y}\right)$, $\left(\frac{\dot{L}}{L}\right)$ y $\left(\frac{\dot{K}}{K}\right)$ representan las tasas de crecimiento del producto, el trabajo y el capital, respectivamente.

$\frac{\dot{A}}{A}=\frac{\dot{Y}}{Y}-\alpha \frac{\dot{L}}{L}-(1-\alpha) \frac{\dot{K}}{K}$

La definición más utilizada de la PTF es la parte del producto no explicada por la cantidad de insumos empleados en la producción (Comin, 2008). Es decir, aquella que representa variaciones de la cantidad del producto no explicadas por variaciones de la cantidad de capital o trabajo. No obstante, Barro (1999, p. 135), quien revisa la literatura, señala que si bien este residuo se suele ver como una medida del progreso tecnológico, puede relacionarse con desembolsos en ciencia y tecnología, políticas públicas y otros factores. Estas posibles interpretaciones dependen del marco de análisis, es decir, de los parámetros adicionales que se incluyan en el modelo.

Para calcular la PTF de un país se toman las series temporales del PIB como producto $(Y)$, de la cantidad de capital como $(K)$, del número de ocupados como $(L)$, y de $(\alpha)$ como participación del trabajo

${ }^{2}$ Sin embargo, este es un resultado al que llega después, cuando introduce los datos, igual que Cobb y Douglas (1928). 
en el producto. Esto último debido a que, como señala Barro (ibíd., p. 120), es impráctico calcular la elasticidad del producto con respecto al trabajo, y resulta más fácil suponer que a los factores se les pagan sus productividades marginales, de modo que $(\alpha)$ sería simplemente el promedio de la proporción del producto que recibe el trabajo y $(1-\alpha)$ la proporción promedio del producto que recibe el capital en ese periodo. Se toman los promedios para que la participación de cada factor sea constante.

\section{CONTROVERSIAS SOBRE LA AGREGACIÓN Y LA IDENTIDAD CONTABLE}

La función Cobb-Douglas y su uso extendido han suscitado varias controversias relacionadas con el problema de la agregación y de la identidad contable que hay detrás de la función. Felipe y McCombie (2005 y 2013) hacen una revisión bibliográfica muy completa de este tema. Lo que se presenta a continuación es un resumen de esa literatura para ilustrar la discusión.

Primero se debe mencionar que hay dos grandes críticas a la función Cobb-Douglas y al modelo de Solow. Por un lado, la crítica a la idea de que es posible definir teóricamente una función de producción agregada por dos razones que poco se discuten en la actualidad: la agregación de las funciones microeconómicas y la agregación del capital. Por el otro, la crítica a la noción de funciones de producción agregadas como transformación de una identidad contable.

Comencemos con el problema de la agregación. En lo que respecta a las funciones microeconómicas, en un marco neoclásico cabe preguntar: ¿qué condiciones se deben cumplir para agregar funciones de producción de empresas con diferentes tecnologías? A este respecto Felipe y McCombie (2013, p. 30) citan a Fisher (2005, p. 490) quien plantea que "las condiciones para la agregación son tan rigurosas que hacen que la existencia de funciones de producción agregada no ocurra en la economía real". Y que, excepto bajo rendimientos constante a escala, es improbable que existan funciones de producción, y que incluso bajo este supuesto no es posible agregar el capital físico heterogéneo ni el producto.

La agregación del capital también ha sido tema de gran controversia, aunque es determinante para definir una función de producción. De acuerdo con Cohen y Harcourt (2003), la controversia comenzó con el artículo de Joan Robinson (1953-1954) que preguntó en qué unidades se mide el capital y puso en cuestión la manera tradicional de agregar capitales heterogéneos, un tema central en el debate entre los dos Cambridge, junto a las controversias sobre la noción de equi- 
librio y la falta de robustez de los modelos. Aunque en opinión de Cohen y Harcourt, esos debates son cruciales, se dejaron de lado con la llegada de los nuevos modelos de crecimiento en los años ochenta, y la mayoría de los economistas hoy los desconocen. No obstante, el problema de la agregación y las controversias de Cambridge son de vital importancia en el debate sobre el cálculo de la productividad, como se verá más adelante.

El segundo grupo de críticas hacen referencia a las funciones de producción agregadas, en particular la Cobb-Douglas, como transformación de una identidad contable. El primer trabajo que planteó ese tipo de críticas es el de Phelps Brown (1957), quien señaló que esta función se basa en una identidad contable, pues la elasticidad del producto del trabajo y su participación en el producto son en realidad "dos lados de la misma moneda".

$\mathrm{Su}$ argumento se puede resumir de la siguiente manera ${ }^{3}$. Si $\left(V_{i}\right)$ es el valor monetario del producto y $\left(J_{i}\right)$ el valor monetario del capital, la elasticidad del producto del trabajo en la función Cobb-Douglas se define como $(\alpha)$ siempre que se cumplan las condiciones de primer orden.

$V_{i}=A_{0} L_{i}^{\alpha} J_{i}^{\beta}$

$\left(\frac{\partial V_{i}}{\partial L_{i}}\right)\left(\frac{L_{i}}{V_{i}}\right)=\alpha$

Partiendo de la identidad contable del producto acorde con el pago de los factores, y derivando el producto con respecto al trabajo se obtiene que el resultado es igual a los salarios. $Y$ al multiplicar a ambos lados de la identidad por $\left(\frac{L_{i}}{V_{i}}\right)$ se llega a que la productividad marginal del trabajo multiplicada por este factor es igual a la participación del trabajo en el producto, definida como $a_{i}$.

$V_{i} \equiv w_{i} L_{i}+r_{i} J_{i}$

$\left(\frac{\partial V_{i}}{\partial L_{i}}\right) \equiv w_{i}$

$\left(\frac{\partial V_{i}}{\partial L_{i}}\right)\left(\frac{L_{i}}{V_{i}}\right) \equiv w_{i}\left(\frac{L_{i}}{V_{i}}\right) \equiv a_{i}$

Por tanto, al igualar (9) y (12) se llega a que la llamada elasticidad del producto respecto al trabajo $(\alpha)$ es realmente la participación del trabajo en el producto $\left(a_{i}\right)$. Es decir, aun sin ninguno de los supuestos de la teoría neoclásica que pretende explicar la distribución del producto

${ }^{3}$ Por claridad de la exposición en adelante se utiliza la notación de Felipe y McCombie (2013). 
entre capital y trabajo a partir de las productividades marginales de cada factor, se llega al mismo resultado. Esto muestra que la función Cobb-Douglas, más que una función que refleje las condiciones técnicas de una firma o de una economía, es la transformación de una identidad contable.

Un trabajo que sigue la misma vía es el de Simon y Levi (1963). Estos autores señalan que dada la identidad contable en la función Cobb-Douglas, el uso de datos reales siempre estará muy cerca de indicar que se trata de una función con rendimientos constantes a escala. Es decir, que al usar datos reales del capital y el trabajo la suma de las elasticidades del producto con respecto a cada factor ( $\alpha$ y $\beta)$ tendrá un valor muy cercano a 1 .

Un resumen de este argumento, tal como lo presentan Felipe y McCombie (2013, pp. 53-54), se puede expresar así. Partiendo de la función Cobb-Douglas de la ecuación (8), dividiéndola por $V_{i}^{\prime}=A_{0} L_{i}^{\prime \alpha} J_{i}^{\prime \beta}$ (expresión que es un valor de referencia, como p. ej. una firma representativa) y usando logaritmos a ambos lados de la ecuación se obtiene:

$\ln \frac{V_{i}}{V_{i}^{\prime}}=\alpha \ln \frac{L_{i}}{L_{i}^{\prime}}=\beta \ln \frac{J_{i}}{J_{i}^{\prime}}$

Y tomando la aproximación de la serie de Taylor, según la cual $\ln \frac{X_{i}}{X_{i}} \approx\left(\frac{X_{i}}{X_{i}}-1\right)$, se llega a la siguiente expresión.

$V_{i}=\left(\alpha \frac{V_{i}}{L_{i}^{\prime}}\right) L_{i}+\left(\beta \frac{V_{i}}{J_{i}^{\prime}}\right) J_{i}+(1-\alpha-\beta) V_{i}^{\prime}$

Por otro lado, al tomar la identidad contable $V_{i} \equiv w_{i} L_{i}+r_{i} J_{i}$ y aplicar la aproximación de la serie de Taylor se llega a la siguiente transformación.

$V_{i}=w_{i} L_{i}+r_{i} J_{i}+(1-a-(1-a)) V_{i}^{\prime}$

Comparando (14) y (15) se encuentran dos conclusiones importantes. Primera, al igual que las interpretó Phelps, $(\alpha)$ y $(\beta)$ son simplemente las participaciones del trabajo y el capital en el producto. Segunda, $(1-\alpha-\beta) V_{i}^{\prime}$ debe ser igual a 0 ; por tanto, el análisis de los datos siempre sugerirá rendimientos constantes a escala.

Otro trabajo importante en esta discusión, pero que dirige su crítica al modelo de Solow, es el de Shaikh (1974), quien examina la identidad contable usando datos de series de tiempo. Shaikh toma una serie de datos aleatorios de producto y capital per cápita de un plano, donde escribe HUMBUG (que traduce "farsa"), los usa para hacer una regresión del modelo de Solow y encuentra que tiene un 
ajuste casi perfecto. Con una crítica punzante y certera, demuestra que siempre que los datos las participaciones del capital y el producto sean relativamente constantes el modelo tendrá un muy buen ajuste.

Lo que es esencial aquí es que el análisis neoclásico no es adecuado para comprobar su teoría de la distribución del producto. Como señalan Felipe y McCombie (2013, p. 50), el problema es que esta teoría no se puede validar usando de funciones de producción con valores monetarios del capital y el producto, pues para calcular la elasticidad de cada factor con respecto al producto se deberían usar valores físicos del producto y el capital. Así, a partir de $Y=A L^{\alpha} K^{\beta}$ se podría calcular valor de $\alpha$ y $\beta$ y luego comprobar la hipótesis de que estos valores se aproximan a la distribución del producto de los factores. Pero cuando se usan valores monetarios suponiendo que son proxis de los valores físicos, la estimación de $V_{i}=A_{0} L_{i}^{\alpha} J_{i}^{\beta}$ siempre produce buen ajuste porque es una trasformación de una identidad contable. $\mathrm{Y}$ en este caso los valores de $(\alpha)$ y $(\beta)$ no son más que las participaciones del trabajo y el capital en el producto $(a)$ y $(1-a)$.

Es necesario señalar que así como este análisis es válido para la función Cobb-Douglas también lo es para las demás funciones de la teoría neoclásica. La función CES, la Translog, y la Transformación Box-cox son transformaciones de la identidad contable, como demuestran Felipe y McCombie (2013, pp. 84-91), quienes muestran que solo son ampliaciones de la función Cobb-Douglas y, por tanto, que sus buenos ajustes no validan esta teoría.

Si el modelo de Solow se basado en una identidad contable, igual que la función Cobb-Douglas, cabe preguntar ¿qué se halla cuando se calcula la PTF? Shaikh (1974) dio una respuesta, que siguiendo a Felipe y McCombie (2013), se puede resumir así. Partiendo nuevamente de la identidad contable, usando logaritmos a ambos lados de la ecuación y derivándola parcialmente con respecto al tiempo para transformarla en tasas de crecimiento de cada una de las variables, se obtiene:

$$
\begin{aligned}
& V_{t} \equiv w_{t} L_{t}+r_{t} J_{t} \\
& \frac{\dot{V}_{t}}{V_{t}} \equiv \frac{\dot{W}_{t}}{W_{t}}\left(a_{t}\right)+\frac{\dot{L}_{t}}{L_{t}}\left(a_{t}\right)+\frac{\dot{r_{t}}}{r_{t}}\left(1-a_{t}\right)+\frac{\dot{J}_{t}}{J_{t}}\left(1-a_{t}\right)
\end{aligned}
$$

Teniendo en mente que hay una diferencia crucial entre el uso de valores físicos del capital y el producto y el uso de sus valoraciones monetarias, el modelo de Solow se puede expresar con esta notación, 
como en la ecuación (8). Además, se puede hacer la misma transformación que ya se hizo a la identidad contable.

$$
\frac{\dot{V}_{t}}{V_{t}}=\frac{\dot{A}_{t}}{A_{t}}+\alpha \frac{\dot{L}_{t}}{L_{t}}+\beta \frac{\dot{J_{t}}}{J_{t}}
$$

Pero como ya se demostró, $(\alpha)$ y ( $\beta$ ) son las participaciones del capital $\mathrm{y}$ el trabajo en el producto, que se pueden remplazar por $(a) \mathrm{y}(1-a)$, y la función se representa así:

$\frac{\dot{V}_{t}}{V_{t}}=\frac{\dot{A}_{t}}{A_{t}}+\frac{\dot{L}_{t}}{L_{t}}\left(a_{t}\right)+\frac{\dot{J_{t}}}{J_{t}}\left(1-a_{t}\right)$

Comparando (17) y (19) se observa que el crecimiento de la PTF es en realidad un promedio ponderado de la variación del salario y la tasa de ganancia promedio de la economía, cuyo ponderador es la participación del trabajo en el producto.

$\frac{\dot{A}_{t}}{A_{t}} \equiv \frac{\dot{w}_{t}}{w_{t}}\left(a_{t}\right)+\frac{\dot{r}_{t}}{r_{t}}\left(1-a_{t}\right)$

Shaikh muestra que la PTF no se puede equiparar a la productividad. $\mathrm{Y}$ aunque Solow pretenda encontrar en qué medida el crecimiento de una economía se puede explicar por la variación de los factores de producción, y qué parte por factores diferentes, como la productividad, este residuo no cumple su objetivo.

\section{EL CÁLCULO DE LA PRODUCTIVIDAD Y SU INTERPRETACIÓN}

La literatura privilegia la PTF como proxy de la productividad porque toma en cuenta los distintos factores y no solo uno de ellos. E1 problema es que, como ya se mostró, no refleja lo que se espera de ella, pues parte de una función de producción agregada que es una simple transformación de una identidad contable. Cabe, entonces, preguntar cómo calcular la productividad. Esta sección presenta las posibilidades existentes, sus interpretaciones y sus limitaciones.

Mark (1972), el Manual de Productividad de la OCDE (2001) y el Manual de Productividad de la Oficina Nacional de Estadísticas (ONS) del Reino Unido (2007) resumen los diferentes conceptos de productividad y su interpretación. Según estos trabajos, y al parecer es un consenso en la literatura, la noción más general de productividad es la de un aumento de la cantidad física de producto con la misma cantidad física de alguno de sus insumos, cualquiera que sea (trabajo, capital, energía, etc.), o una combinación de estos. Hay entonces tantos conceptos de productividad como factores de producción. 
E1 Manual de la OCDE incluye el siguiente cuadro, que resume las diferentes medidas de productividad.

Resumen de las principales medidas de productividad

\begin{tabular}{|c|c|c|c|}
\hline \multirow{2}{*}{$\begin{array}{l}\text { Medición del } \\
\text { producto }\end{array}$} & \multicolumn{3}{|c|}{ Medición de los insumos } \\
\hline & Trabajo & Capital y trabajo & $\begin{array}{l}\text { Capital, trabajo } \\
\text { e insumos inter- } \\
\text { medios (ener- } \\
\text { gía, materiales, } \\
\text { servicios) }\end{array}$ \\
\hline Producto bruto & $\begin{array}{l}\text { Productividad la- Productividad del } \\
\text { boral (basada en capital (basada en } \\
\text { producto bruto) producto bruto) }\end{array}$ & $\begin{array}{l}\text { PMF Capital- } \\
\text { trabajo (basada } \\
\text { en producto } \\
\text { bruto) }\end{array}$ & $\begin{array}{l}\text { Productividad } \\
\text { multifactorial de } \\
\text { CTEMS }\end{array}$ \\
\hline Valor añadido & $\begin{array}{cc}\text { Productividad } & \text { Productividad del } \\
\text { laboral (basada } & \text { capital (basada en } \\
\text { en valor añadido) } & \text { valor añadido) }\end{array}$ & $\begin{array}{l}\text { PMF Capital- } \\
\text { trabajo (basada } \\
\text { en valor añadido) }\end{array}$ & - \\
\hline & $\begin{array}{l}\text { Medidas de productividad de un } \\
\text { solo factor }\end{array}$ & $\begin{array}{r}\text { Medidas de produ } \\
\text { torial }(\end{array}$ & $\begin{array}{l}\text { ctividad multifac- } \\
\text { PMF) }\end{array}$ \\
\hline
\end{tabular}

Fuente: OCDE (2001, p. 13).

El modo de calcular la productividad se diferencia entonces teniendo en cuenta uno o varios insumos. En el primer grupo se encuentran la productividad laboral y la productividad del capital. En el segundo, la productividad multifactorial, teniendo en cuenta únicamente capital y trabajo, o estos dos insumos y otros que se pueden añadir. La productividad multifactorial es sinónimo de productividad total de los factores y, como ya se demostró, no refleja la variación de la productividad. Examinemos las interpretaciones y los alcances de la productividad del capital y de la productividad del trabajo.

Empecemos por la productividad del capital, que se mide como la cantidad de producto por unidad de capital. Teóricamente, esta medición tiene un problema conceptual. Recordando la controversia de Cambridge, no tiene sentido hablar de productividad del capital, debido a la circularidad en la determinación del valor del capital (Robinson, 1953-1954, p. 81). Es decir, no es posible establecer el valor de la cantidad de capital, pues para ello es necesario establecer primero la tasa de ganancia, y esta, a su vez, depende del valor de la cantidad de capital.

Sin embargo, en el mundo real se puede determinar el valor de la cantidad de capital a partir de sus precios de mercado. El problema práctico es que el valor monetario del producto por unidad de valor monetario de capital no refleja cuán productiva es una tecnología en uso. Lo que indica esta medida es cuán intensivo es el uso de la cantidad de capital para obtener una cantidad monetaria de producto, 
pues una mejor calidad de los bienes de capital implica un aumento del capital utilizado con respecto al producto y, por tanto, se refleja en una caída de la "productividad del capital" (OCDE, 2001, p. 17).

Para aclarar este punto supongamos que para producir un valor monetario $\mathrm{A}$ de producto, se usa un valor monetario $\mathrm{B}$ de capital en un periodo $t$. La relación producto-capital es entonces $\mathrm{A} / \mathrm{B}$ en este periodo. Ahora supongamos que se introduce una nueva tecnología más intensiva en capital, que requiere menos trabajo para obtener la misma cantidad de producto y más rentable para el dueño del capital. Ser más intensiva significa que la proporción del valor monetario del capital es mucho mayor, es decir que $\mathrm{B}<\mathrm{C}$, donde $\mathrm{C}$ es la cantidad de capital en el periodo $t+1$. Si el precio del producto se mantiene igual en ambos periodos, el valor monetario del producto en $t+1$ será $\mathrm{A}$, con la diferencia de que esta nueva tecnología es más rentable para el capital. La relación producto-capital en el periodo $t+1$ será $\mathrm{A} / \mathrm{C}$. Al comparar la llamada productividad del capital del periodo $t$ con la de $t+1$ se encuentra que disminuyó, lo que sería absurdo interpretar como una caída de la productividad, pues hubo una mejora tecnológica.

No obstante, medir la evolución del producto por cantidad de capital puede ser útil según lo que se intente analizar. Puede servir para analizar la productividad de una firma en un periodo específico donde no se ha modificado el capital que utiliza. En este caso puede revelar cuál ha sido el cambio en la productividad debido a un nuevo arreglo del proceso productivo que hace más eficiente la producción o mostrar si ha habido un aumento en la intensidad del trabajo, como plantea el Manual de productividad de la ONS (2007, p. 7).

La productividad laboral, por su parte mide la cantidad de producto por unidad de trabajo ${ }^{4}$. Las unidades de trabajo se pueden medir de dos maneras. La primera mide la cantidad de producto por persona empleada y la segunda, la cantidad de producto por hora trabajada. La productividad se calcula entonces partiendo de la relación producto por trabajador o por hora trabajada $(q)$.

$q_{L}=\frac{V}{L} \circ q_{b}=\frac{V}{H}$

Usando logaritmos naturales en ambos lados de la ecuación y derivando con respecto al tiempo se llega a que la tasa de crecimiento del producto con respecto al trabajo -la variación de la productividad-es

${ }^{4}$ Esta medida no se debe confundir con la productividad laboral que emplean autores neoclásicos como Espinosa y Vaca (2013), definida a partir de la PTF. 
igual a la tasa de aumento del producto menos la tasa de aumento de los trabajadores empleados o del número de horas trabajadas.

$\frac{\dot{q}_{L}}{q_{L}}=\frac{\dot{V}}{V}-\frac{\dot{L}}{L} \mathrm{o} \frac{\dot{q}_{H}}{q_{H}}=\frac{\dot{V}}{V}-\frac{\dot{H}}{H}$

La productividad laboral capta las variaciones del producto sin los problemas de la productividad del capital. Aunque se puede pensar que la productividad laboral no es del todo válida porque en su medición no se incluye el capital. Pero no es así, pues si con un número de trabajadores dado se introduce una nueva y mejor tecnología, representada por el capital, es evidentemente que la cantidad de producto por trabajador aumenta, es decir la productividad laboral se incrementa. De hecho, todo cambio en el proceso de producción que lo haga más eficiente se refleja en este cálculo, porque la reducción de la cantidad de trabajo empleada para obtener una misma cantidad de producto es, por definición, lo que hace más productiva a una sociedad o empresa. En últimas, esta es una medida adecuada debido a que el capital tiene vida propia y es incapaz de reproducirse a sí mismo.

Para interpretar la productividad laboral hay que tener en cuenta varios aspectos. Primero, no refleja necesariamente un aumento de la intensidad del trabajo, pues el producto puede aumentar por una mejora tecnológica manteniendo constante el trabajo, y así este indicador reflejaría el cambio técnico.

Segundo, Nordahaus (2002, pp. 9-10) y Balakrishnan (2004, p. 1468-9), presentan una serie de posibles efectos que capta el cálculo de la productividad y que no reflejan un cambio técnico: como el efecto Deninson y el efecto Baumol. El primero hace referencia a cambios en la composición del producto entre sectores. Por ejemplo, cuando se traslada capital de un sector agrícola poco productivo a un sector industrial altamente productivo, la productividad agregada aumenta aunque no haya incrementos de productividad en cada sector.

Por otro lado, el efecto Baumol, llamado también enfermedad de los costos, se refiere al cambio de la participación en el producto de dos sectores, cuando en uno de ellos hay mejoras tecnológicas y el otro permanece con una productividad relativamente constante. En este caso el precio relativo del producto del primer sector disminuye con respecto al del segundo, y viceversa. El ejemplo clásico es el de un cuarteto de cuerdas con respecto a los bienes tecnológicos. Per ejemplo mientras que el precio relativo de los computadores disminuye, el precio relativo de un concierto del cuarteto aumenta. Pues en el caso del cuarteto la productividad no una varía, por cuanto siempre se necesitan cuatro personas que ensayen y toquen, mientras que la 
producción de un computador es cada vez más barata debido al avance tecnológico. De modo que el cálculo de la productividad de toda la economía no refleja totalmente el cambio tecnológico, sino que se limita al cambio en la participación de los sectores en el producto.

Tercero, la medición de la productividad depende de la relación entre la variación del producto total y la variación del número de ocupados. En un periodo de recesión productividad laboral puede disminuir o aumentar, dependiendo cuánto se vean afectadas la producción y las ventas en relación con el desempleo. Si el desempleo aumenta (el número de ocupados disminuye) más que la caída del crecimiento del producto, la variación de la productividad será positiva. Si el producto cae más que el número de ocupados, la variación de la productividad será negativa.

\section{LA BRECHA}

Después de revisar la discusión sobre el cálculo de la PTF y las interpretaciones y alcances de la productividad laboral, analizamos los datos del salario mínimo colombiano en el periodo 2001-2016 para establecer el posible impacto de esta discusión en los aumentos de dicho salario. Se analiza este periodo debido a que se dispone de datos de productividad laboral, por número de ocupados y por horas trabajadas, con respecto al $\mathrm{PIB}^{5}$.

En primer lugar, cuando se examinan la variación anual de la productividad laboral y la PTF resaltan dos cosas. Primera, su comportamiento es muy similar, y se puede afirmar que su variación está correlacionada. Segunda, en la mayoría de los años la PTF ha sido menor que la productividad laboral.

La diferencia acumulada entre la productividad laboral y la PTF es del 17,3\% en el periodo. Esto significa claramente que, para negociar el salario mínimo, la variación de la productividad que se ha considerado es significativamente menor que la que se observa realmente en la economía colombiana. Así, el salario ha aumentado menos de lo que se podía esperar.

Este hecho se demuestra en forma contundente cuando se compara la variación del salario mínimo real con la productividad laboral. La gráfica 4 muestra un índice donde el año 2000 toma un valor de 100.

${ }^{5}$ Hay dos cálculos de productividad laboral disponibles. Uno realizado por el DNP con la metodología de la OCDE (2001) para calcular la productividad laboral por persona ocupada. E1 otro, de productividad laboral por número de horas trabajadas, es realizado directamente por la OCDE. 
Gráfica 3

Productividad laboral y PTF, Colombia 2001-2016

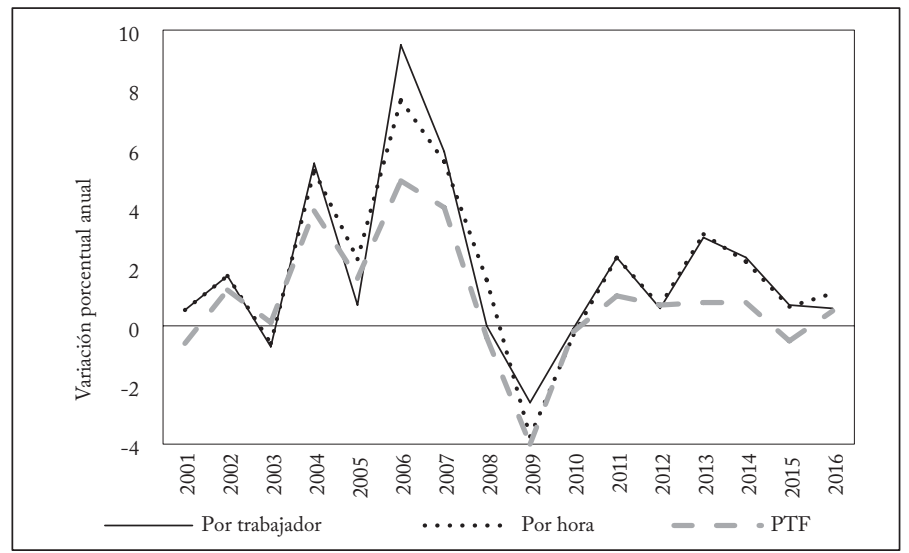

Fuente: DNP y OCDE, elaboración propia.

Gráfica 4

Índice de productividad laboral y salario mínimo real $(2000=100)$

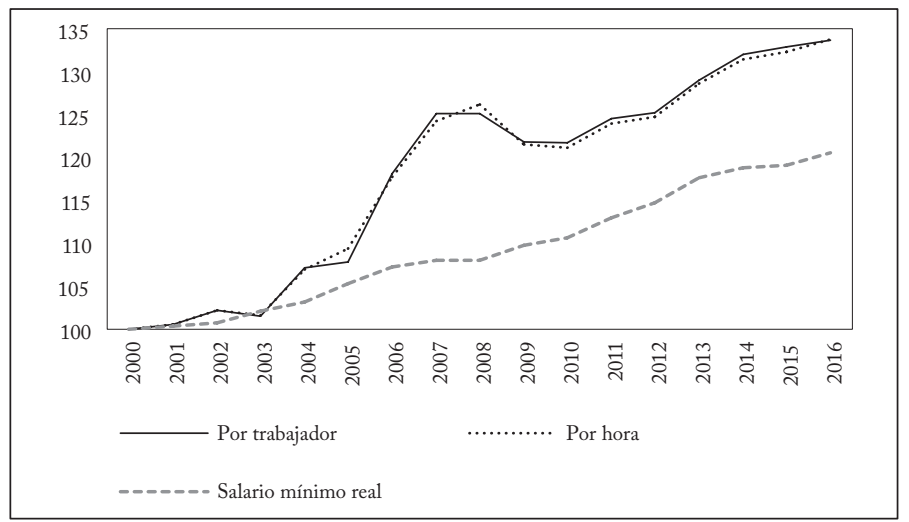

Fuente: DNP y OCDE, elaboración propia.

La gráfica muestra que entre el salario mínimo y la productividad existe una brecha que viene aumentando desde 2004. Esto indica que el salario mínimo del país sigue la misma tendencia que se observa a nivel mundial descrita a comienzos del artículo. En 2016, la brecha acumulada entre el salario mínimo y la productividad llegó al 11\%.

En suma, estos datos muestran que los aumentos del salario mínimo no solo tuvieron en cuenta erróneamente uno de sus criterios, sino que, además, se omitieron los demás criterios señalados por la normatividad colombiana. Por ello, mientras que el crecimiento 
acumulado del salario mínimo real con respecto al de la PTF es superior, debido a la diferencia acumulada del 17,3\% entre la PTF y la productividad laboral este salario muestra en realidad un rezago del $11 \%$ con respecto a esta última.

\section{RELACIÓN ENTRE PTF Y PRODUCTIVIDAD}

Como muestra la gráfica 3, existe una fuerte correlación entre la PTF y la productividad. Cabe preguntar entonces cuál es la razón. Lo primero que se puede pensar es que la PTF es un indicador de productividad, pero ya se demostró que no es así. A continuación veremos que hay otra razón para explicar este comportamiento similar.

Remplacemos la tasa de crecimiento del producto de la ecuación (7) por la de la ecuación (22), con el cambio de notación correspondiente. Reorganizando términos se obtiene que la PTF es igual a la productividad menos la resta de las variaciones del capital $\left(\frac{j}{J}\right)$ y del trabajo $\left(\frac{L}{L}\right)$ multiplicadas por la participación del capital en el producto $(1-a)$. Esto indica que cuando el capital crece más rápido que el trabajo la PTF es menor que la productividad. En el caso contrario, cuando la variación del capital es menor que la del trabajo la PTF es mayor que la productividad.

$$
\begin{aligned}
& \frac{\dot{q}}{q}=\frac{\dot{A}}{A}+\alpha \frac{\dot{L}}{L}+(1-a) \frac{\dot{J}}{J}-\frac{\dot{L}}{L} \\
& \frac{\dot{A}}{A}=\frac{\dot{q}}{q}-(1-a)\left(\frac{\dot{J}}{J}-\frac{\dot{L}}{L}\right)
\end{aligned}
$$

Esta relación se puede corroborar comparando los datos de variación del capital con los del trabajo, y los cálculos de la PTF y de la productividad en sus años respectivos. Esas comparaciones confirman que hay una brecha entre la PTF y la productividad laboral, la cual obedece a que en la mayoría de los años la tasa de crecimiento de la cantidad de capital ha sido mayor que la de la población ocupada.

\section{EL CRECIMIENTO EN LA PRODUCTIVIDAD Y LA DISTRIBUCIÓN DEL INGRESO}

Después de mostrar la evolución de la brecha entre productividad y salario mínimo es posible discutir los efectos de esta brecha en la distribución del ingreso entre capital y trabajo. Aunque primero es necesario mencionar dos críticas adicionales a los aspectos empíricos y epistemológicos de la teoría neoclásica de la distribución del ingreso. Comenzamos por los aspectos empíricos. 
Como se ya se mostró, no existe una comprobación empírica de la teoría, pues como la función Cobb-Douglas es una transformación de una identidad contable, sus buenos ajustes no comprueba la teoría. Y puede ser imposible comprobarla debido a los problemas de agregación del capital señalados por Robinson. Para comprobarla habría que tomar $(\alpha)$ y $(\beta)$ calculadas a partir de mediciones del capital físico, y compararlas con las participaciones reales de cada factor en el producto. El problema es que, como se toma el valor monetario del capital y del producto, lo que así se calcula son las participaciones del trabajo y del capital en el producto, lo cual es una tautología.

Parte del trabajo de Felipe y McCombie (2006) busca comprobar si existe esa relación entre productividades marginales e ingresos de los factores. Mediante una serie de modelos y datos aleatorios del producto, el capital y el trabajo en términos físicos, encuentran que no existe razón alguna para que las elasticidades marginales del producto con respecto al trabajo y al capital guarden relación con los pagos a cada factor. Es decir, que $(\alpha)$ y $(\beta)$ sean iguales o, al menos, cercanos a $(a)$ y $(1-a)$.

También se crítica el marco teórico neoclásico que se adopta para explicar la distribución del ingreso. Keen (2011) resume esas críticas y muestra los problemas de suponer que el ingreso de los factores, capital y trabajo, corresponde a su productividad marginal.

Primero muestra que si se eliminan algunos supuestos del modelo neoclásico no se encuentra relación alguna entre productividad marginal del trabajo y salarios. Luego señala que un problema central es que la teoría neoclásica supone que el mercado de trabajo se comporta como cualquier otro mercado, y que todo aumento del precio (el salario) del producto (la fuerza de trabajo) eleva la oferta de los trabajadores. Y argumenta que puede ocurrir lo contario: que ante caídas del salario estén dispuestos a trabajar más, y que aumentos de salarios incentiven a trabajar menos y a tener más tiempo libre. Esto implica que, en el marco neoclásico, la curva de oferta en este mercado tendría una pendiente negativa y no positiva, como se supone, lo que pone en cuestión su noción de rendimientos decrecientes a escala. Recuerda, además, que Sraffa, usando modelos en los que no estaban dadas la tasa de ganancia ni la cantidad de capital, demostró que:

no solo no había una relación uniforme entre la tasa de ganancia y la cantidad de capital, sino que la dirección de la causalidad era opuesta a la que deseaban los economistas. En vez de que la tasa de ganancia dependa de la cantidad de capital, la cantidad medida de capital realmente depende de la tasa de ganancia. (Keen, 2011, p. 155) 
Así, los rendimientos del capital pueden aumentar o disminuir a medida que este factor se añade a la producción.

No hay entonces una relación consistente entre productividad del factor e ingreso de los factores. En cambio, la distribución del ingreso entre salarios y ganancias es en gran medida independiente del sistema de producción. La distribución del ingreso es un fenómeno social. (ibíd., 156)

En suma, no hay un marco teórico robusto ni evidencia empírica que implique que los ingresos de los factores guardan una relación con su productividad marginal. La distribución del ingreso entre capital y trabajo responde a razones ajenas al mecanismo de mercado.

Esto implica que cuando mejora la productividad no existe un mecanismo automático que aumente los ingresos del trabajo. Surge entonces una disputa distributiva entre capital y trabajo. Como señala Marx (1977), esta es una característica del sistema capitalista, donde los propietarios de los medios de producción intentan elevar su tasa de ganancia introduciendo tecnologías que aumenten la productividad, es decir, el producto por trabajador. Así pueden aumentar la plusvalía relativa. Es decir, el nuevo producto añadido debido a las mejoras tecnológicas queda en un campo de disputa que no se resuelve en el mercado. Por ello, la dinámica de la acumulación y los arreglos institucionales que se tejan a su alrededor determinan esta distribución.

Lapavitsas se refiere así a este punto:

Marx consideró la productividad del trabajo como un factor fundamental en la determinación de la generación de plusvalía (ganancias), y, por tanto, de la competencia capitalista y de la tasa de ganancia. Si la duración de la jornada laboral viene dictada por las prácticas institucionales y el equilibrio de la lucha de clases (incluido el sistema legal), y los salarios nominales están dados, los capitalistas podrían mejorar la competitividad mediante el aumento de la productividad o mediante la intensificación del trabajo. De esas dos opciones, el aumento de la productividad es de lejos la más sostenible a mediano y largo plazo. El método para aumentar la productividad a largo plazo lo constituye, a su vez, el desarrollo y la introducción sistemática de nuevas tecnologías. (Lapavitsas, 2016, p. 235)

De modo que el aumento de los salarios al ritmo de la productividad es parte de la disputa entre el capital y el trabajo. Y se podría pensar que el aumento de salarios a la par de la productividad significa que todos los frutos del desarrollo tecnológico van al trabajo. Pero lo cierto es que aumentar los salarios, entre ellos el salario mínimo, al ritmo de la productividad genera una participación constante del capital y del trabajo en el producto. Para demostrarlo basta partir de la ecuación (12), con el cambio de notación correspondiente, sabiendo además que la participación en el producto $(a)$ es justamente $(\alpha)$. 
$W=a\left(\frac{V}{L}\right)$

Remplazando $\left(\frac{V}{L}\right)$ por $(q)$ y reordenando términos se obtiene la expresión (26). Esta indica que para que la participación del trabajo en el producto (a) sea constante, así como la del capital $(1-a)$, los salarios $(w)$ deben crecer al ritmo de la productividad laboral $(q)$.

$a=\frac{w}{q}$

Gráfica 5

Salario mínimo como porcentaje del PIB per cápita

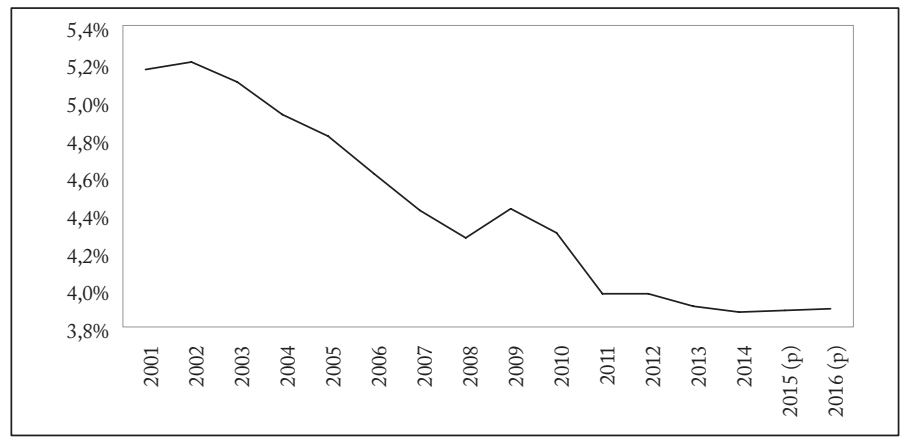

Fuente: Banco de la República, elaboración propia.

Esto implica que cuando los salarios crecen a menor ritmo que la productividad, a los trabajadores les corresponde una proporción cada vez más pequeña del producto. Esta tendencia es bastante clara en el caso del salario mínimo colombiano. La gráfica 5 muestra que el salario mínimo como porcentaje del PIB per cápita cayó un 24,6\% en 2001-2016. Es decir, mientras que la economía creció, el salario de los trabajadores que ganan el mínimo no aumentó al mismo ritmo.

Por último, el hecho de que los salarios crezcan al ritmo de la productividad no implica que haya una distribución justa o equitativa del producto. Solo garantiza que la distribución entre el capital y el trabajo se mantenga constante, y con las mismas diferencias salariales entre trabajadores. Como vimos, esa diferencia en los niveles de ingresos no es determinada por el mercado sino por diputas políticas y sociales, y por el curso del proceso de acumulación capitalista.

\section{CONCLUSIONES}

La normatividad colombiana incluye la productividad como criterio para aumentar el salario mínimo. En los últimos 16 años esta se ha 
calculado a partir del modelo de Solow, cuyo residuo es la PTF. El problema es que muchos autores demuestran que este residuo no se puede interpretar como productividad. Además, la comparación del crecimiento real del salario mínimo con la productividad laboral muestra una brecha del 11\% en el periodo 2001-2016, calculada por horas trabajadas y por número de trabajadores.

Sin embargo, existe una relación contable entre la PTF y la productividad laboral: la primera es mayor o menor que la segunda dependiendo de que la tasa de variación de la cantidad de capital sea mayor o menor que la tasa de crecimiento de la cantidad de trabajo. Por ello, en este periodo existe una brecha del 17,3\% entre la PTF y la productividad laboral, brecha que a su vez es la fuente del rezago del salario mínimo con respecto a los criterios establecidos para aumentarlo. Esta brecha se traduce en una pérdida de participación de los trabajadores que ganan un salario mínimo en el ingreso nacional, como indica el salario mínimo como porcentaje del PIB per cápita, que se redujo en un $24,6 \%$. Esto significa que así el salario mínimo haya tenido incrementos en términos reales, no han sido iguales a los de la tasa de crecimiento de la economía, lo cual aumenta la desigualdad en el país.

Quedan por estudiar varios temas relacionados con la productividad y el aumento de salarios, incluido el del salario mínimo. En particular, un tema muy discutido: la relación entre salario mínimo, desempleo e informalidad. Parte de la literatura, en especial la de carácter neoclásico, afirma que el salario mínimo genera desempleo e informalidad y que, por tanto, aumentos del salario empeoran la situación del mercado laboral. Entre los diversos trabajos que se refieren al caso colombiano desde esta perspectiva cabe destacar los de Núñez y Bonilla (2001), Hernández y Díaz (2006) y Bautista (2013). No obstante, Álvarez, Santacruz et al. (2012, pp. 28-29) reseñan varios trabajos que no encuentran una relación concluyente entre incremento del salario mínimo y aumento de la tasa de desempleo. Esto significa que aún hay mucho trabajo por hacer y que el enfoque de la brecha de productividad puede arrojar más luces sobre el tema, como sugiere Shaikh (2016).

\section{REFERENCIAS BIBLIOGRÁFICAS}

Álvarez, A., Santacruz, A. et al. (2012). El salario mínimo en Colombia. Una perspectiva comparada y recomendaciones. En S. Moreno y A. Pulido (eds.), Tercerización laboral en el sector financiero y evolución salarial en Colombia (pp. 17-73). Cali: Impresos Diaman. 
Balakrishnan, P. (2004). Measuring productivity in manufacturing sector. Economic and Political Weekly, 39(14/15), 1465-1471.

Barro, R. J. (1999). Notes on growth accounting. Journal of Economic Growth, 4(2), 119-137.

Bautista, L. F. (2013). Efectos de un incremento del salario mínimo en el desempleo y la inflación Colombia 2001-2012. [https://repository. javeriana.edu.co/bitstream/handle/10554/14852/BautistaPinerosLuisFernando2013.pdf; sequence=1].

Cobb, C. W. y Douglas, P. H. (1928). A theory of production. The American Economic Review, 18(1), 139-165.

Cohen, A. y Harcourt, G. (2003). Whatever happened to the Cambridge capital theory controversies? Journal of Economic Perspectives, 17(1), 199-214.

Comin, D. (2008). Total factor productivity. En S. Derlauf y L. Blume (eds.), The new palgrave dictionary of economics, $2 .^{a}$ ed. (pp. 329-330). Hampshire, UK: Palgrave Macmillan.

Cortés, P. J. (2010). Política de salarios mínimos en Colombia. Una lectura entre la teoría y la realidad. Equidad y Desarrollo, 13, 119-126.

Douglas, P. H. (1976). The Cobb-Douglas production function once again: Its history, its testing, and some new empirical values. Journal of Political Economy, 84(5), 903-916.

Espinosa, O. A. y Vaca, P. A. (2013). PTF y productividad laboral en Colombia 1970-2010. Econografos, 35, 1-47.

Felipe, J. y McCombie, J. (2005). La función de producción agregada en retrospectiva. Investigación Económica, 44(253), 43-88.

Felipe, J. y McCombie, J. (2006). The tyranny of the identity: Growth accounting rivisited. International Review of Applied Economics, 20(3), 283-299.

Felipe, J. y McCombie, J. (2013). The aggregate production function and the measurement of technical change. Northampton: Edward Elgar.

Fisher, F. M. (2005). Aggregate production functions - a pervasive, but unpersuasive, fairytale. Eastern Economic Journal, 31(3), 489-491.

Hernández, G. y Pinzón, E. (2006). El efecto del salario mínimo sobre el empleo y los ingresos. Archivos de Economía, 316, 1-40.

Keen, S. (2011). Debunking economics - revised and expanded edition: The naked emperor dethroned? Londres: Zed Books.

Lapavitsas, C. (2016). Beneficios sin producción. Cómo nos explotan las finanzas [2013]. Madrid: Traficantes de sueños.

Mark, J. A. (1972). Meanings and measures of productivity. Public Administration Review, 32(6), 747-753.

Marx, C. (1977). El Capital [1867], vol. I. Bogotá: Fondo de Cultura Económica.

McGrattan, E. R. y Prescott, E. C. (2012). The great recession and delayed economic recovery: A labor productivity puzzle? En L. E. Ohanian, J. B. Taylor y I. J. Wright (eds.), Government policies and the delayed economic tecovery (pp. 115-154). Stanford, Ca: Hoover Institution Press.

Nordhaus, W. D. (2002). Alternative methods for measuring productivity growth including approaches when output is measured with 
chain indexes, [http://www.econ.yale.edu/ nordhaus/homepage/ welf_062402.pdf].

Núñez, J. y Bonilla, J. D. (2001). ¿Quiénes se perjudican con el salario mínimo en Colombia? Coyuntura Social, 24, 87-110.

OECD. (2001). Measuring productivity: OECD Manual: Measurement of aggregate and industry-level productivity growth. París: OECD.

ONS. (2007). The ONS productivity handbook. A statistical overview and guide. Nueva York: Palgrave Macmillan.

Phelps B., E. H. (1957). The meaning of the fitted Cobb-Douglas function. The Quarterly Journal of Economics, 71(4), 546-660.

Rivera, D. Y. (2014). Salario mínimo en Colombia. Econógrafos, 56, 1-28.

Robinson, J. (1953-1954). The production function and the theory of capital. The Review of Economic Studies, 21(2), 81-106.

Shaikh, A. (1974). Laws of production and laws of algebra: The humbug production function. The Review of Economics and Statistics, 56(1), 115-120.

Shaikh, A. (2016). Capitalism. Competition, conflict, crises. Nueva York: Oxford University Press.

Simon, H. A. y Levy, F. K. (1963). A note on the Cobb-Douglas function. The Review of Economic Studies, 30(2), 93-94.

Solow, R. M. (1956). A contribution to the theory of economic growth. The Quarterly Journal of Economics, 70(1), 65-94.

Solow, R. M. (1957). Technical change and the aggregate production function. The Review of Economics and Statistics, 39(3), 312-320.

Valle, A. (1991). Productividad: las visiones neoclásica y marxista. Investigación Económica, 50(198), 45-69.

Wolff, R. D. (2016). The wages of global capitalism. En M. L. Palmeri y D. Dallavalle (eds.), Capitalisms crisis deepens essays. Essays on the global economic meltdown 2010-2014 (pp. 68-72). Chicago: Haymarket Books. 